\title{
Une lettre de Jean Marx à Jacques Maritain
}

\section{Michel Fourcade}

\section{(2) OpenEdition}

\section{Journals}

Édition électronique

URL : http://journals.openedition.org/rbnu/965

DOI : $10.4000 /$ rbnu.965

ISSN : 2679-6104

\section{Éditeur}

Bibliothèque nationale et universitaire de Strasbourg

\section{Édition imprimée}

Date de publication : 1 mai 2017

Pagination : 94-99

ISBN : 9782859230661

ISSN : 2109-2761

\section{Référence électronique}

Michel Fourcade, « Une lettre de Jean Marx à Jacques Maritain », La Revue de la BNU [En ligne], 15 | 2017, mis en ligne le 01 novembre 2019, consulté le 15 décembre 2020. URL : http://

journals.openedition.org/rbnu/965; DOI : https://doi.org/10.4000/rbnu.965

\section{(C) $10(0$}

La Revue de la BNU est mise à disposition selon les termes de la Licence Creative Commons Attribution - Pas d'Utilisation Commerciale - Partage dans les Mêmes Conditions 4.0 International. 


\section{Cherjacques,}

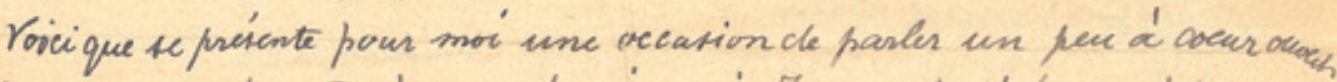
et lihment avee toi gráa à une entermise amie. Ie ne said ti tu avair requ la lettre quefe t'avais adrenée par cliffer an cours de I'hives de $19 \mathrm{f}$ en

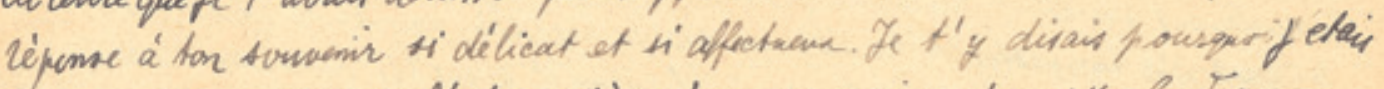
preu tenti, en depit de l'atmosphire p'on y respire. de quittu la Fanee

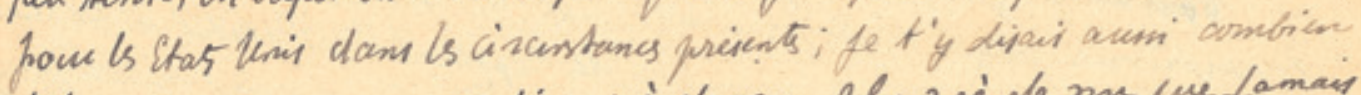

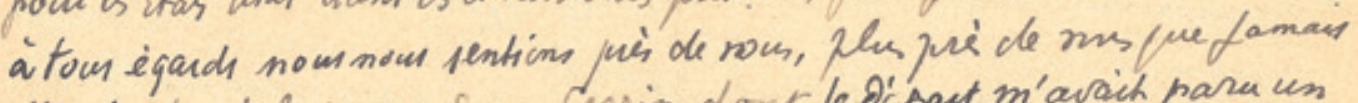
I'arau changé le pawore jcan Gerrin dont ledipact m'avich para un peu impre dent en zajon de son e'tat de santé de te dise nos amitiú profingy. How arm $\mathrm{Pu}$ arec une admization et we énotion de toute nothe

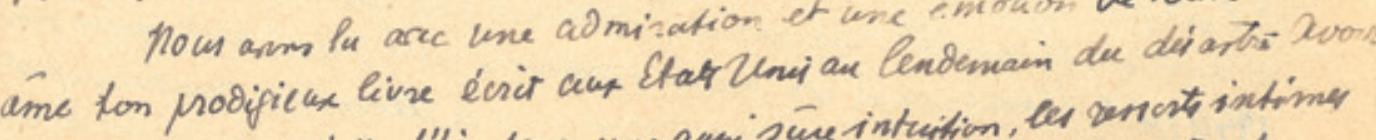

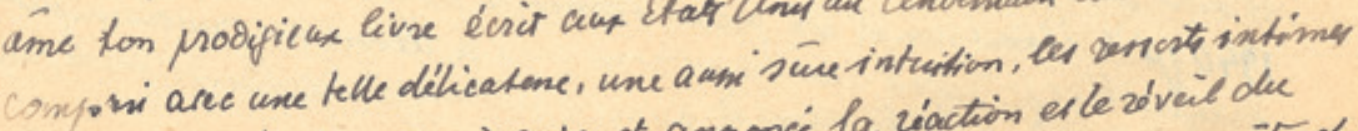
du tommes el des chores, anoir priru et annonci la viaction erle vóveit due

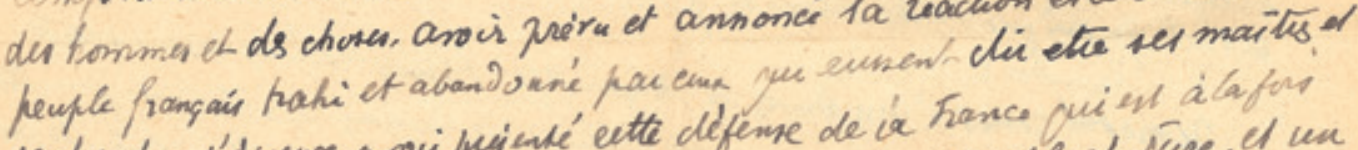

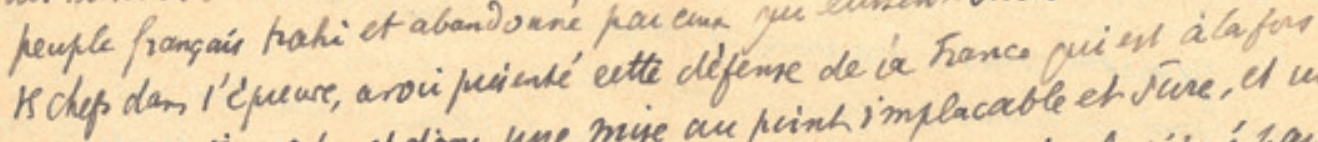
unc accusation sobe et digne, une mise au pinh implacable et jüre, dt un

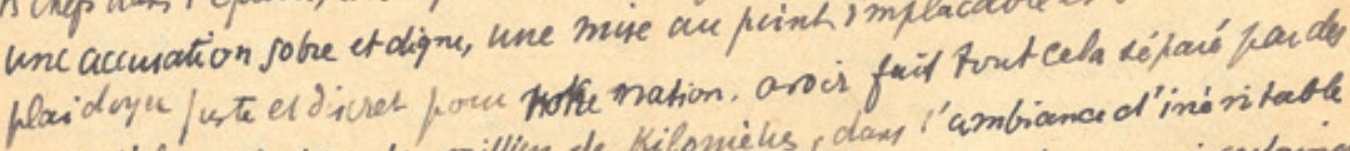

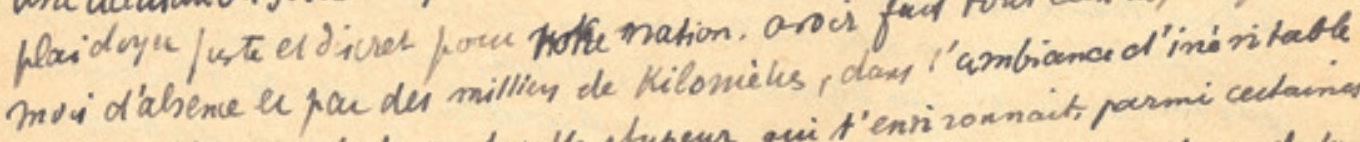
incomput hemion at de nature le stupeur qui t'enrizonnaits parmi cectainas

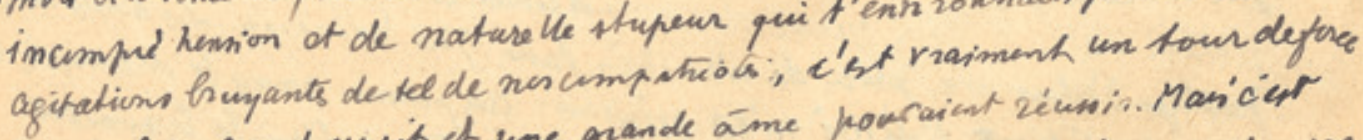
que teul an grand epprit et une grande áme povraicat zéusii. Mariciefr triqui amas pante le timajnage conte lequel zion ne parait a ne pourra Mévaloir.

Ye ne saci situ a au courant de cette impumion frofonde caurée dans

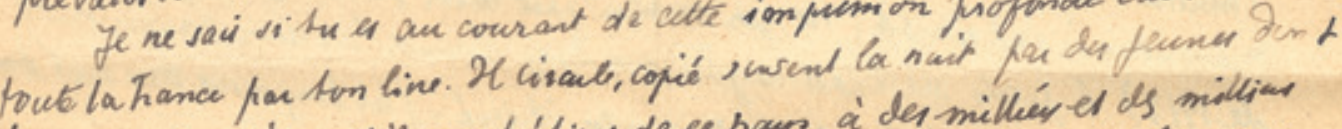
becuunes ont ter meithen chétions de ce pays, à der miltiés el ds milliar

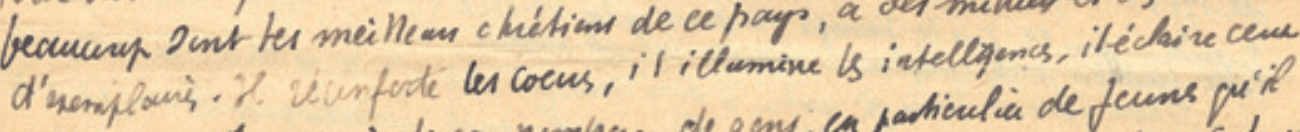

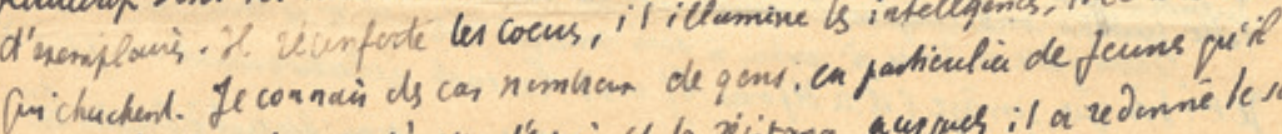

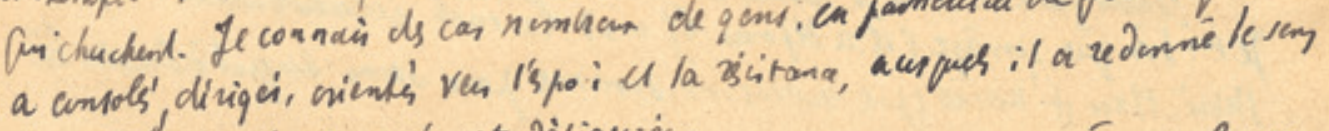
de ceter hane abanjonncé et dèfiguree.

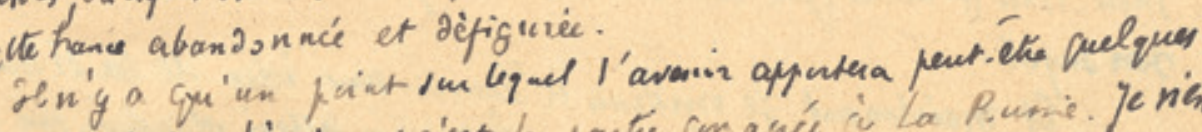

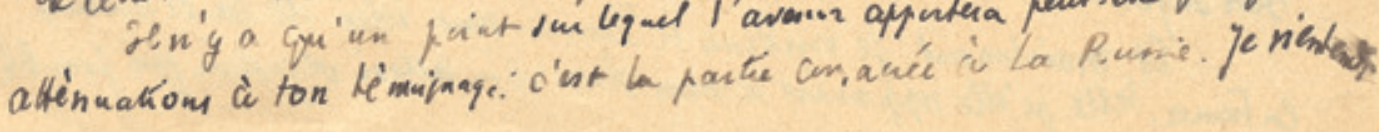




\section{Une lettre de Jean Marx à Jacques Maritain}

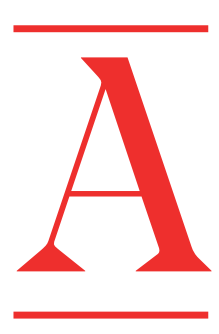

cte de deuil et d'espérance, méditant les causes profondes de la débâcle française de juin 1940 et risquant un jugement sur les premiers mois d'Occupation, À travers le désastre, publié par Jacques Maritain à New York en janvier 1941, fut le premier grand livre des Français exilés pendant la guerre. Aussitôt traduit en anglais, en allemand, en portugais, en espagnol, très lu chez les Français libres de Londres au cours de l'été 1941, l'ouvrage s'introduit aussi en Europe occupée où il devient le premier et l'un des principaux « bestsellers » de la littérature clandestine. Souvent qualifié de "prophétique ", l'essai joue ainsi un rôle essentiel dans l'éveil des consciences et favorise beaucoup la maturation politique des premières Résistances, intérieures comme extérieures ${ }^{1}$. À tous ceux déjà collectés, la lettre que nous publions ici (issue du fonds Maritain de la Bibliothèque nationale et universitaire de Strasbourg $^{2}$ ) ajoute un précieux témoignage : non datée, elle a été rédigée à Lyon, fin juin 1942 sans doute, comme l'indiquent l'allusion à la mort le 17 avril du physicien Jean Perrin, en exil lui aussi à New York, puis la référence quelques paragraphes plus loin à l'actualité de la bataille de Tobrouk. Comme le précise son carnet, Maritain l'a reçue le 28 août avec d'autres courriers de métropole : « Ce que Marx dit de l'accueil fait à mon livre en France est bouleversant ».
L'auteur, Jean Marx (1884-1972), comme sa sœur Suzanne, était resté lié aux Maritain depuis leur jeunesse étudiante, dreyfusarde et bergsonienne : Raïssa venait de la raconter dans le premier tome de ses Grandes amitiés, publié également à New York et reçu lui aussi en France occupée comme un mince rayon de lumière. C'est l'un de ces Israélites français que la défaite et la législation antisémite du régime de Vichy ont chassé brutalement de la fonction publique. Chartiste (entré et sorti major, 1908-1912), auteur d'une thèse sur L'Inquisition en Dauphiné (1914) puis pensionnaire de l'École française de Rome à l'époque de Mgr. Duchesne, cet érudit enseignait ses spécialités - les religions primitives de l'Europe, la littérature celtique, la légende arthurienne, le cycle du Graal -, à la V ${ }^{\mathrm{e}}$ section de l'École des Hautes Études depuis 1925. Parallèlement à cette carrière savante, ses engagements lors de la Première Guerre mondiale l'avaient aussi rattaché au Quai d'Orsay, jusqu'à succéder à Giraudoux en 1933 comme chef du service des Euvres françaises à l'étranger : durant tout l'entre-deux-guerres, il s'est donc consacré au développement et au rayonnement des instituts, des chaires et des lycées français du monde entier $^{3}$. Une fois la guerre déclarée à l'automne 1939, c'est lui qui a organisé la tournée de conférences de Maritain aux États-Unis, pour sensibiliser une opinion encore profondément isolationniste aux enjeux idéologiques et spirituels du conflit ${ }^{4}$. Embarqués à Marseille le 4 janvier 1940 sur l'Exochorda, les Maritain se 
retrouvent bloqués par la défaite à New York et Jacques ne pourra revenir en France qu'en novembre 1944.

Par-delà le désastre, à l'heure où il reçoit cette lettre, le philosophe vient de terminer deux nouveaux essais consacrés à la refonte des idéaux démocratiques : "munitions idéologiques ", Les Droits de l'homme et la loi naturelle puis Christianisme et démocratie accentuent son audience interalliée et s'introduisent également en France. Son appartement new yorkais de la $5^{\mathrm{e}}$ Avenue est un point de ralliement de "l'Europe en exil », et grâce à des courriers comme celui que nous publions, l'un des lieux les mieux informés des réalités souterraines des pays occupés. Associé dès l'origine au projet d'une université en exil, placée lors de son inauguration le 14 février 1942 sous le signe de la France libre, il en est le président officieux puis officiel après la mort en mars 1943 d'un autre ami de Jean Marx, Henri Focillon. Particulièrement attentif à la persécution des Juifs, et prenant conscience très précocement du projet génocidaire, Maritain dénonce aussi dans ses messages radiodiffusés la complicité de Vichy dès les premières rafles de ce même été 1942. Décrivant de son côté une opinion se remettant peu à peu en état de mobilisation résistante, la lettre de Jean Marx pourra certes sembler optimiste : dans l'immédiat, la chape de l'Occupation s'alourdit, tandis que l'invasion allemande de novembre 1942 achève de vassaliser la «zone libre ». Paul-René Marx, le frère resté à Paris et que Jean espérait ici protégé par son âge, disparaîtra à Auschwitz en février 1943. Les dénonciations de la presse collaboratrice et les recherches de la Gestapo conduiront bientôt Jean et Suzanne Marx à se cacher en Saône-et-Loire, avec l'aide des F.F.I. locaux. Mais l'aventure à laquelle ils survivront sera aussi spirituelle : appelé au chevet de Suzanne Marx quelques mois après la Libération, Maritain lui donnera le baptême ; il sera également le parrain de Jean en 1948.

\section{Michel Fourcade}

Cher Jacques ${ }^{5}$,

Voici que se présente pour moi une occasion de parler un peu à cœur ouvert et librement avec toi grâce à une entremise amie. Je ne sais si tu avais reçu la lettre que je t'avais adressée par clipper au cours de l'hiver de 1940 en réponse à ton souvenir si délicat et si affectueux. Je t'y disais pourquoi j'étais peu tenté, en dépit de l'atmosphère qu'on y respire, de quitter la France pour les États-Unis dans les circonstances présentes ; je t'y disais aussi combien à tous égards nous nous sentions près de vous, plus près de vous que jamais. J'avais chargé le pauvre Jean Perrin, dont le départ m'avait paru un peu imprudent en raison de son état de santé, de te dire nos amitiés profondes.

Nous avons lu avec une admiration et une émotion de toute notre âme ton prodigieux livre écrit aux États-Unis au lendemain du désastre. Avoir compris avec une telle délicatesse, une aussi sûre intuition, les ressorts intimes des hommes et des choses, avoir prévu et annoncé la réaction et le réveil du peuple français trahi et abandonné par ceux qui eussent dû être ses maîtres et ses chefs dans l'épreuve, avoir présenté cette défense de la France qui est à la fois une accusation sobre et digne, une mise au point implacable et sûre, et un plaidoyer juste et discret pour notre nation, avoir fait tout cela séparé par des mois d'absence et par des milliers de kilomètres, dans l'ambiance d'inévitable incompréhension et de naturelle stupeur qui t'environnait, parmi certaines agitations bruyantes de tel de nos compatriotes, c'est vraiment un tour de force que seul un grand esprit et une grande âme pouvaient réussir. Mais c'est toi qui auras porté le témoignage contre lequel rien ne pouvait et ne pourra prévaloir. 
Je ne sais si tu es au courant de cette impression profonde causée dans toute la France par ton livre. Il circule, copié souvent la nuit par des jeunes dont beaucoup sont les meilleurs chrétiens de ce pays, à des milliers et des milliers d'exemplaires. Il réconforte les cœurs, il illumine les intelligences, il éclaire ceux qui cherchent. Je connais des cas nombreux de gens, en particulier de jeunes, qu'il a consolés, dirigés, orientés vers l'espoir et la résistance, auxquels il a redonné le sens de cette France abandonnée et défigurée.

Il n'y a qu'un point sur lequel l'avenir apportera peut-être quelques atténuations à ton témoignage : c'est la partie consacrée à la Russie. Je n'entends pas l'absoudre, ni absoudre ses amis de chez nous, de leurs graves torts. Mais nous connaissons maintenant les circonstances atténuantes du passé, et l'héroïque combat du présent. Nos gouvernants n'avaient pas su comprendre qu'une fois de plus le seul moyen de galvaniser le peuple des champs et des usines, c'était de lui donner la certitude qu'il combattait pour sa liberté et pour la Liberté, avec tous les peuples libres. Eux s'acharnaient, conduits par une caste incompréhensive et déjà moralement conquise par le fascisme, à vider la guerre de tout contenu idéologique : du coup ils se montraient incapables d'entraîner la France dans un élan de bataille comme ils avaient été incapables de la préparer sur le terrain militaire et technique. Pour moi, en quittant les Affaires étrangères, il avait été entendu que je donnerais mon cours à l'École des Hautes Études. Le manque de courage des fonctionnaires de l'Instruction publique en face de l'application du statut, et le fait que ma fonction principale était aux Affaires étrangères et disparaissait automatiquement du fait de ce statut, amenaient ma mise à la retraite que je ne regrette point. Car elle pose le problème qui me concerne, après vingt ans de service que tu connais et dont un des ministres passagers de l'Instruction publique me disait en août 1940 qu'ils étaient ceux d'un grand serviteur de l'État (sic), dans son intégralité et je préfère vraiment n'avoir rien à devoir au gouvernement actuel. Je sais que d'autres seront là pour reconstruire et réparer. Je serai toujours prêt à répondre à leur appel.

Je me suis remis à mes études de Moyen Âge : depuis de longues années je méditais un travail sur les éléments celtiques des romans arthuriens. J'ai trouvé à Lyon tous les textes essentiels, et j'ai réuni les éléments d'un livre qui, je crois, sera neuf et vrai sur cette matière. Ma sœur qui a supporté tous ces temps très durs avec un courage et une dignité que tu devines, partage mon exil lyonnais. J'allais rentrer à Paris quand au mois d'août le ministre de l'Instruction publique d'alors me pria de n'en rien faire. J'ai trouvé ici des amis excellents dont la délicate fidélité m'est précieuse : au premier rang les Massigli, les Wladimir d'Ormesson, les Fila et tout le groupe du Figaro. Dans le travail et l'amitié soutenus par l'espoir plus fort que jamais en dépit de déceptions momentanées inévitables (ne pas oublier que nous sommes au printemps de 1918), le poids de l'exil ne pèse pas trop lourd. Car nous savons qu'il prendra fin et que les temps ne sont plus éloignés. J'ai toujours cru en l'Amérique, elle justifie ma confiance ancienne et totale.

Mon frère a tenu à rester à Paris malgré mes efforts et ceux de nos amis. Je sais bien que son âge (il a dépassé 65 ans) le met relativement un peu à l'abri. Mais j'avoue que nous ne sommes pas sans inquiétude à son sujet. 
Et maintenant je voudrais te donner une idée de la situation en France, telle qu'elle apparaît à mon observation aussi objective que possible. Je ne t'apporterai pas grand-chose de neuf, mais le résultat d'expériences nombreuses et précises.

Certes ce pays a été mal conduit et mal gouverné. Mais plus encore la guerre y a été mal préparée et l'armée mal dirigée par un commandement incapable de comprendre la guerre moderne, ses méthodes et ses engins. Les témoignages là-dessus dès maintenant sont écrasants. Les paroles dont tu as avec une divination merveilleuse démonté les faux prestiges ont réussi momentanément, dans l'amertume cuisante de la défaite, à éluder les responsabilités et à humilier la nation dans une fausse pénitence qui n'avait rien de viril, de spirituel ni de profond. En même temps la coalition d'une bourgeoisie déchue, égoïste et avide et d'une bande d'aventuriers recevant des inspirations étrangères depuis de longues années, qui rêvaient depuis de longues années aussi d'une reprise du pouvoir, s'est jetée sur cette occasion. C'est le mot terrible d'un ancien (et glorieux) combattant à l'un de mes amis : « J'ai fait deux guerres ; dans l'une j'aurai vu une victoire contre l'étranger ; dans l'autre une victoire contre l'intérieur ». À la base de la défaite il y avait le sentiment de la supériorité de l'ennemi, l'acceptation de son hégémonie morale, à l'abri de laquelle la bourgeoisie croyait (bien sottement) trouver la consolidation de ses privilèges, la sauvegarde de ses capitaux et la répression des mouvements ouvriers dont notre régime aura en fait décuplé la vigueur et la violence.

Mais ces faux prestiges empêchèrent des réactions nécessaires. Beaucoup d'honnêtes gens épris d'ordre, de catholiques ayant souffert (comme nous tous) de la bêtise agressive du régime défunt se rallièrent au mot d'ordre. Ainsi s'étendit la chape de plomb, le linceul de bêtise, de conformisme bassement bienpensant, de défaitisme satisfait, d'humiliation masochiste dans lesquels on allait s'efforcer d'ensevelir la France.

Mais tous ces gens s'étaient en fait trompés sur toute la ligne. Ils attendaient de l'Allemagne des procédés généreux : ils obtinrent la mise au pillage et la menace du démembrement. Ils escomptaient la défaite de l'Angleterre et l'Angleterre tint bon stoïquement. $\mathrm{Ne}$ l'oublions pas au jour de Tobrouk. Ils voyaient le fascisme et le totalitarisme imposant par leur victoire leur idéologie à l'Europe et ils allaient assister à un étonnant réveil des idées et des sentiments démocratiques rénovés et d'un internationalisme recréé. Ils croyaient à la localisation du conflit, et l'intervention des États-Unis allait donner à la guerre un caractère mondial et intercontinental tandis que l'agression contre la Russie et la résistance de l'URSS saisissaient à la gorge les masses ouvrières de tous les pays.

Alors, la gestion d'une administration incapable se joignant à la tyrannie d'une occupation implacable, se produisit cette réaction héroïque de la France prophétiquement annoncée par toi et dont tu n'avais jamais douté. On s'accorde à penser qu'en dépit de tous les efforts, la zone occupée entière, à l'exception de quelques milliers de profiteurs, et dans la zone libre toute la masse ouvrière, la presque totalité des paysans, la quasi-unanimité des fonctionnaires attendent impatiemment le jour qui les replacera dans la guerre et où ils pourront s'exprimer librement. 
On assiste ici à un réveil, inintelligible pour la prétendue élite si peu consciente de nos vraies traditions, des sentiments républicains dans le peuple de France qui a le sentiment qu'on lui a volé son idéal. On me signale par exemple que dans un département du centre de la France le buste de la République retiré des mairies est couvert de fleurs dans chaque commune rurale par des centaines de paysans. Le mouvement est irrésistible et partout attesté. Les catholiques y ont heureusement leur rôle et leur part : beaucoup de Jocistes ${ }^{6}$, de catholiques sociaux, de prêtres et d'étudiants sont là pour faire oublier Baudrillart et les abus de certains bien-pensants que tu connais mieux que moi.

Que l'Amérique fasse confiance au peuple de France, mais point à ceux qui rêvent d'une médiation entre Franco et Salazar pour sauver l'hégémonie allemande sur l'Europe et le grand capitalisme français.

Ce qu'il faudrait c'est ne pas manquer la paix. Tu assistes, je crois, aux États-Unis, à un mouvement sur le terrain national dans un sens de corporatisme vraiment et noblement démocratique, sur le terrain international à une action dans le sens du fédérationnisme et de l'internationalisme rénovés. Mais si l'Allemagne n'est pas durement traitée dans ses chefs, ses meneurs et son élite, elle recommencera dans dix ans, même au lendemain d'une expérience révolutionnaire et communiste.

Voilà des idées un peu rapides. Que j'aimerais à en parler à loisir avec toi. J'aimerais savoir aussi ce que vous faites, Raïssa, Véra et toi. Nous pensons souvent à vous. J'ai vu le livre de R. grâce au charmant Guillemin ${ }^{7}$ mais je ne l'ai point. Nous avons été bien touchés, Suzanne et moi, des quelques lignes qu'elle nous a consacrées. Vous êtes de ceux qu'il est bien naturel d'aimer.

Écris-moi si tu le peux par une voie sûre. Mon adresse est 6 rue Gasparin à Lyon. Si tu empruntes la voie habituelle soumise à l'inévitable censure, porte-moi le souvenir de Jacques Henry ${ }^{8}$.

Nous vous embrassons.

Jean

NOTES

1- Cf. M. Fourcade, « Jacques Maritain inspirateur de la résistance ", in Cahiers J. Maritain, n 32, 1996, p. 14-57 et $\mathrm{n}^{\circ} 64,2012$, p. 21-45. Voir également les notices consacrées à l'ouvrage et à son auteur dans le Dictionnaire de la France libre, Robert Laffont, coll. « Bouquins », 2010.

2- Voir La Revue de la BNU, $\mathrm{n}^{\circ}$ 11, rubrique « Nouvelles acquisitions patrimoniales ».

3- Cf. M. Degros, « Jean Marx», in Bulletin de l'École des chartes, 1973, vol. 131, 2, p. 684-687

4- Cf. F. Michel, « Jacques Maritain pendant la 'drôle de guerre’ (septembre 1939-juin 1940) », in Cahiers J. Maritain, $\mathrm{n}^{\circ} 59,2009$, p. 4-15, et la correspondance avec Jean Marx publiée en annexe, p. 39-57.

5- Nous retranscrivons ici le texte sans modifications autres que d'éventuelles corrections orthographiques. De même, on a ici ou là rajouté un signe de ponctuation (Marx en utilise très peu), afin de faciliter la lecture (note de l'éditeur).

6- On appelait ainsi les membres de la Jeunesse ouvrière chrétienne (J. O. C.).

7- Il s'agit du critique littéraire Henri Guillemin (1903-1992), qui fit lire à Jean Marx le premier tome des Grandes amitiés, publié par Raïssa à New York en 1941 aux éditions de la Maison française.

8- C'est sous ce dernier pseudonyme que Maritain correspond avec ses amis restés en France occupée. 